\title{
SERVICIOS WEB 2.0 EN EL PORTAL UNICA
}

\author{
Web 2.0 services in the UNICA site
}

Rigo Grimaldos* y Anny Paz ${ }^{* *}$

\section{RESUMEN}

El presente trabajo tuvo como objetivo determinar el uso de los servicios web 2.0 en el portal de la Universidad Católica Cecilio Acosta (UNICA) por parte de la institución, ubicada en la ciudad de Maracaibo, Venezuela. Los aportes teóricos se sustentaron en O’Reilly (2007), Lévy (2004), Rheingold (2004), Surowiecki (2005), Cobo (2007a), entre otros autores. La investigación procede de un proyecto adscrito al Decanato de Investigación y Postgrado de la UNICA, fue de tipo descriptiva, se aplicó una guía de observación para recolectar información del portal web de la UNICA y los servicios 2.0 a los que está asociado como las plataformas para redes sociales verticales YouTube, Instagram y SoundCloud, y las plataformas para redes sociales horizontales Facebook y Twitter. Entre los hallazgos más relevantes se evidenció el uso exclusivo de los servicios de social networking para la interacción con la comunidad universitaria, sin incluir otros servicios que procuran la generación de conocimiento. Se concluye que la UNICA emplea los servicios web 2.0 en su portal de forma limitada y poco estratégica, con un carácter meramente informativo que incluye temas específicos de la universidad y la iglesia católica, lo que podría obstaculizar la gestión del conocimiento como proceso propio de las universidades del siglo XXI.

Palabras clave: servicios web 2.0, TIC, gestión del conocimiento, virtualidad.

RECIBIDO: Junio 2019

ACEPTADO: Julio 2019

\section{ABSTRACT}

This investigation aimed to determine the use of web 2.0 services in the Catholic University Cecilio Acosta (UNICA) site by the institution, located in Maracaibo city, Venezuela. The theoretical contributions were supported by O'Reilly (2007), Lévy

\footnotetext{
* Magíster en Comunicación y Desarrollo, mención Tecnologías de la Información y la Comunicación para el Desarrollo Humano. Licenciado en Comunicación Social, mención Desarrollo Social. Docente de la Universidad Católica Cecilio Acosta (UNICA), adscrito a la Facultad de Ciencias de la Comunicación Social. Maracaibo, Venezuela. Correo electrónico: rigogrimaldos@gmail.com

${ }_{* *}^{*}$ Magister Scientiarum en Ciencias de la Comunicación, mención Gerencia de la Comunicación. Docente-investigadora de la Universidad del Zulia (LUZ) / Universidad Católica Cecilio Acosta (UNICA). Maracaibo, Venezuela. Correo electrónico: annypazbaptista@gmail.com
} 
(2004), Rheingold (2004), Surowiecki (2005), Cobo (2007a), among other authors. The investigation comes from a project attached to the UNICA Research and Postgraduate Deanery, was descriptive, an observation guide was applied to collect information from the UNICA website and the 2.0 services to which it is associated as the platforms for vertical social networks YouTube, Instagram and SoundCloud, and platforms for horizontal social networks Facebook and Twitter. Among the most relevant findings was the exclusive use of social networking services for interaction with the university community, without including other services that seek to generate knowledge. It is concluded that UNICA uses web 2.0 services in its site in a limited and non-strategic way, with a purely informative nature that includes specific topics of the university and the catholic church, which could hinder the knowledge management as process of $21 \mathrm{st}$ century universities.

Keywords: web 2.0 services, ICT, knowledge management, virtuality.

\section{Introducción}

Casi culminados dos decenios del siglo XXI, la sociedad continúa su transitar por contextos emergentes sumergidos en los avances tecnológicos digitales que determinaron para la nueva era cambios, quizás, más complejos que los acontecidos tras el paso de la era agraria a la era industrial. Esta vez, los cambios emanan de otro tipo de tecnología centrado en nuevos códigos y vías de comunicación que permitieron la manipulación de datos e información en formatos digitales para promover posteriormente la evolución del conocimiento, las denominadas Tecnologías de la Información y la Comunicación (TIC) determinan avances tan importantes para el mundo que, actualmente, constituyen uno los principales pilares de la sociedad y sus diversos ámbitos.

La evolución de la Word Wide Web a una Web 2.0 posiciona a la Internet como un medio de comunicación matriz que alcanza niveles de interactividad nunca registrados por otros medios creados, lo que consolida una Sociedad del Conocimiento cuya premisa es la construcción de conocimiento en el hombre a partir de su contacto con datos e información dispuestos en el ciberespacio o en dispositivos tecnológicos digitales.

El presente artículo es el resultado de un proyecto de investigación adscrito al Decanato de Investigación y Postgrado de la Universidad Católica Cecilio Acosta 
(UNICA), en la ciudad de Maracaibo, Venezuela. El trabajo tiene como objetivo determinar el uso de los servicios web 2.0 en el portal de la Universidad Católica Cecilio Acosta (UNICA) por parte de la institución. Los resultados de este estudio de caso se presentarán y analizarán desde los datos obtenidos durante el primer semestre de 2018 (enero-junio), para responder a las interrogantes de investigación del proyecto:

1. ¿Cuáles son los servicios web 2.0 presentes en el portal de la UNICA?

2. ¿Cuál es el uso de los servicios web 2.0 presentes en el portal de la UNICA por parte de la institución?

\section{Metodología}

Para este estudio, descriptivo, se realizó una observación en el ciberespacio concretamente en el portal de la Universidad Católica Cecilio Acosta (www.unica.edu.ve), además de los servicios web 2.0 que se vinculan desde el mismo portal en un período comprendido entre los meses enero y junio de 2018 (6 meses). Los servicios web 2.0 quedaron concentrados en las cuentas de usuario de la UNICA (unicaeduve) en las plataformas para redes sociales YouTube (14 publicaciones),

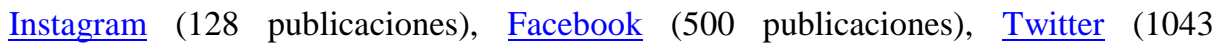
publicaciones) y SoundCloud (0 publicaciones). Se aplica la técnica de investigación exclusivamente en plataformas para redes sociales debido a que no se encontró otro tipo de servicio web 2.0 en el portal como los de contenido, los de organización inteligente de información o los de aplicaciones mashup ${ }^{1}$.

La observación se realizó en dos fases: la primera consistió en explorar el portal de la UNICA para identificar los servicios web 2.0 compartidos a través de botones, íconos u otros que enlazan con plataformas que ofrecen este tipo de servicio, seguidamente, otra fase se centró en el monitoreo de las plataformas para redes sociales

\footnotetext{
${ }^{1}$ Aplicaciones web híbridas (término traducido al español). Este tipo de aplicación ofrece un servicio web que combina el contenido de varias fuentes.
} 
presentes en el portal de la UNICA con las que la institución crea comunidad virtual a través de sus cuentas de usuario.

Los datos obtenidos fueron registrados en una guía de observación semiestructurada, que permitió posteriormente integrar una base de datos para el análisis cuantitativo y cualitativo de la información total. La recolección y el análisis se realizaron en la ciudad de Maracaibo, Venezuela.

\section{Fundamentación teórica}

\section{De la Web 1.0 a la Web 2.0}

Si bien la Internet presenta su origen a finales de los años 60 tras establecer las primeras redes de comunicación mediante computadores interconectados, es la invención de la Word Wide Web (WWW) por Tim Berners-Lee², en 1989, la que permite esbozar la inminente presencia de este medio de comunicación en diferentes contextos de la sociedad que, a partir de los avances en tecnología digital promovidos por las TIC, genera una revolución durante el último decenio del siglo XX.

La WWW o Web 1.0, como es conocida hoy día, se conformó de un conjunto de documentos conectados entre sí a los que se puede acceder por medio de enlaces hipertextuales que se apoyan en los principios de la Internet (Rojo y Sánchez, 2009). Principios que pretenden constituir una red entre miles de ordenadores con el propósito de intercambiar información y permitir comunicaciones de personas a personas, de personas a ordenadores, y de ordenadores a ordenadores (Ibeas et al, 2000). La web 1.0, también conocida como la web del consumidor de contenidos o servicios, permitió en primera instancia la búsqueda de información, la adquisición de productos o servicios, y la comunicación a través de los correos electrónicos (García, 2010).

Adentrados en el siglo XXI, las TIC determinan un inevitable cambio de paradigma debido a la masificación y el uso generalizado de la Internet. La WWW se transforma de acuerdo a las demandas de sus usuarios y a las aplicaciones que surgen

\footnotetext{
${ }^{2}$ Físico británico. Científico de la computación fundador del Consorcio de la World Wide Web (W3C), creador del URL (Uniform Resource Locator, Localizador Uniforme de Recursos), del HTTP (HyperText Transfer Protocol, Protocolo de Transferencia de HiperTexto) y del HTML (Hyper Text Markup Language, Lenguaje de Marcado de HiperTexto).
} 
para satisfacer sus necesidades, escenario que delinea los principios que permitieron a Tim O'Reilly ${ }^{3}$, en 2004, sustentar teóricamente un nuevo concepto acorde a los contextos que emergían en el nuevo siglo, la Web 2.0 representó una evolución de la Internet y la consolidación del paradigma tecnológico.

La Web 2.0, es el entendimiento colectivo de que una red es la plataforma y que en ella las reglas para los negocios son diferentes, la norma fundamental en esta red es que los usuarios agregan valor al descubrir cómo construir bases de datos y mejorarlas mientras son usadas por más personas (O’Reilly, 2007). El surgimiento de este modelo, denominado igualmente web social, introduce un cambio significativo con respecto al modelo anterior donde el usuario sólo consume contenidos, esta nueva web permite a los usuarios producir contenidos y compartirlos con el mundo (García, 2010).

Es a través de la Web 2.0 que los usuarios adquieren un sentido de pertenencia en la red y se convierten en prosumidores ${ }^{4}$ para materializar las predicciones de Alvin Toffler ${ }^{5}$ en 1970. "La idea de la Web 2.0 ha permitido poner en manifiesto el papel absolutamente clave que desempeñan los usuarios en la configuración del medio. Sin su participación activa, buena parte de los servicios existentes en Internet no tendrían sentido" (Nafría, 2008, p.132).

La Web 2.0 se compone de unas características básicas:

a) Es dinámica: los contenidos se actualizan constantemente. b) Son colaborativas: se elaboran por un grupo de personas. c) Son simples e intuitivas. d) Pueden ser utilizadas sin necesidad de instalar nada en un ordenador. e) La web es la plataforma. f) Entorno amigable e interactivo. g) El usuario tiene la capacidad de gestionar: qué, cuándo y cómo publicar (Pardo, 2007; Castaño et al, 2008 citado en Cabero, 2009, p.22).

\footnotetext{
${ }^{3}$ Fundador y presidente de O'Reilly Media. Licenciado en Literatura Clásica, entusiasta de la informática promotor del software libre y el código abierto. Autor del concepto Web 2.0.

${ }^{4} \mathrm{El}$ término prosumidor (prosumer en inglés) es un acrónimo formado de las palabras productor y consumidor, aparece por primera vez en la obra The Third Wave (La tercera ola) de Alvin Toffler, en 1979.

${ }^{5}$ Escritor estadounidense considerado un futurólogo a partir de sus predicciones en obras destacadas como Future Shock (El shock del futuro) de 1970 y The Third Wave (La tercera ola) de 1979, en las que discute acerca de la revolución digital, la revolución de las comunicaciones y la singularidad tecnológica.
} 
Estas características determinan la transición de la Web 1.0 a la Web 2.0, entre las que es posible establecer importantes diferencias:

\section{Cuadro $\mathrm{N}^{0} 1$. Diferencias entre la Web 1.0 y la Web 2.0}

\begin{tabular}{|c|c|c|}
\hline & $\begin{array}{c}\text { Web 1.0 (1993-2003) } \\
\text { Muchas páginas web para ser } \\
\text { vistas a través de un } \\
\text { navegador }\end{array}$ & $\begin{array}{l}\text { Web 2.0 (2003- ) } \\
\text { Multitud de contenidos } \\
\text { compartidos a través de } \\
\text { servicios de alta } \\
\text { interactividad }\end{array}$ \\
\hline Modo & Lectura & Escritura compartida \\
\hline $\begin{array}{l}\text { Minima unidad de } \\
\text { contenido }\end{array}$ & Página & Mensaje - Artículo - Post \\
\hline Estado & Estático & Dinámico \\
\hline Modo visualización & Navegador & Navegador, lector RSS \\
\hline Arquitectura & Cliente - Servidor & Servicio web \\
\hline Editores & Webmasters & Todos \\
\hline Protagonistas & Geeks (gente experta) & Aficionados \\
\hline
\end{tabular}

Fuente: (Castaño et al, 2008, p.33)

\section{Conceptos que emergen alrededor de la Web 2.0}

La constitución de un universo denominado Web 2.0 origina conceptos que, derivados principalmente de sus características, promueven la generación y distribución del conocimiento a través de la evolucionada plataforma. En este contexto, a partir de la generalización de las tecnologías emerge una civilización global con la que el conocimiento individual se transforma en colectivo, un bien público al que todos tienen derecho sin distinciones (Drucker, 2004). Esta noción permite introducir conceptos relacionados con la generación de saberes en entornos caracterizados por la libertad: intercreatividad, inteligencia colectiva, multitudes inteligentes, sabiduría de las multitudes y arquitectura de la participación.

\section{Intercreatividad}

El término intercreatividad lo conceptualiza el creador de la WWW, BernersLee, en su libro intitulado Weaving the Web: The original design and ultimate destiny 
of the World Wide Web ${ }^{6}$ de 1999, en el que describe la creación y desarrollo de una de las invenciones más grandes del siglo XX. Este término, se deriva de las palabras interactividad y creatividad consideradas esenciales para el autor quien percibe desde el momento de su invención el potencial social en la evolución de la Internet.

$\mathrm{La}$ Web es un espacio propicio para que las personas encuentren y creen cualquier tipo de documento, donde pueden interactuar y crear con otras personas para establecer redes de gestión del conocimiento que permitan finalmente hacer o resolver problemas en conjunto, en un proceso de intercreatividad (Berners-Lee, 2000). Desde una perspectiva tecno-social, este término se vincula al uso de las tecnologías en la red y al trabajo colaborativo para compartir conocimiento en el ciberespacio, donde las acciones son recíprocas (Cobo, 2007a).

\section{Inteligencia colectiva}

Pierre Lévy, en 1994, introduce el término en su publicación L'Intelligence collective. Pour une anthropologie du cyberspace ${ }^{7}$, en la que asevera la existencia de un saber colectivo que emana de cualquier lugar habitado por hombre y que éste se puede potenciar cuando es mediado por tecnologías. La inteligencia colectiva es "una inteligencia repartida en todas partes, valorizada constantemente, coordinada en tiempo real, que conduce a una movilización efectiva de las competencias" (Lévy, 2004, p.20).

La inteligencia colectiva está repartida en todas partes porque el conocimiento está en la humanidad, se valoriza constantemente porque antiguos paradigmas la descalifican y restan valor científico, se coordina en tiempo real porque requiere una constante y sólida comunicación que hoy puede potenciarse gracias a las herramientas que ofrece la Web, y se conduce a una movilización efectiva de competencias porque los individuos reconocen sus competencias y la de otros para adquirir una identidad dentro del colectivo (Lévy, 2004). Esta inteligencia representa la capacidad de un

\footnotetext{
${ }^{6}$ Tejiendo la red: El inventor del World Wide Web nos descubre su origen (título en español).

${ }^{7}$ Inteligencia colectiva: Por una antropología del ciberespacio (título en español).
} 
colectivo para colaborar y tomar decisiones que permitan alcanzar sus metas y determinar su rumbo en contextos complejos.

\section{Multitudes inteligentes}

Howard Rheingold desarrolla investigaciones relacionadas con las multitudes inteligentes entre las que destaca su libro Smart mobs: The next social revolution ${ }^{8}$ de 2002, en el que señala la conformación de nuevas redes sociales con características acordes al entorno donde se desarrollan, el ciberespacio. En otros términos, la constitución espontánea de comunidades virtuales según los intereses de quienes la conforman, subculturas que encuentran un nuevo espacio para ser reconocidas.

Las comunidades virtuales se componen de inteligencias emergentes mediante las que se produce un aprendizaje colectivo, con el que se desarrolla habilidades que permiten a cada individuo comprender su rol en contextos que requieren la manipulación de datos e información (Johnson, 2001). Los principios considerados por este autor contribuyen posteriormente con las investigaciones acerca de las multitudes inteligentes, las cuales se establecen por individuos que actúan en conjunto aunque probablemente no lo internalicen tal acción o conozcan a sus pares.

En las multitudes inteligentes, "los miembros de estos grupos cooperan de modos inconcebibles en otras épocas porque emplean sistemas informáticos y de telecomunicaciones muy novedosos que les permiten conectarse con otros sistemas del entorno, así como los teléfonos de otras personas" (Rheingold, 2004, p.18).

\section{Sabiduría de las multitudes}

James Surowiecki, en su publicación The Wisdom of crowds. Why the many are smarter than the few and how collective wisdom shapes business, economies, societies, and nations $^{9}$ de 2004, explica a partir de ejemplos representativos en diversos contextos

\footnotetext{
${ }^{8}$ Multitudes inteligentes: La próxima revolución social (título en español).

${ }^{9}$ Cien mejor que uno. La sabiduría de la multitud o por qué la mayoría siempre es más inteligente que la minoría (título en español).
} 
la importancia que tiene la integración de conocimientos individuales para la toma de decisiones colectivas, además ofrece orientaciones para lograr que los grupos cohesionen y se inclinen por las mejores decisiones frente a elementos que dificultan el proceso.

Las multitudes sabías se caracterizan por aplicar de forma eficaz cuatro componentes esenciales: la diversidad de opiniones, que permite a cada individuo pensar y sustentar información particular; independencia, con la que una opinión personal se desliga de las opiniones de otras personas a su alrededor; descentralización, que promueve la especialización individual a partir de experiencias locales; y agregación, que permite aplicar mecanismos para hacer de los juicios individuales una decisión colectiva (Surowiecki, 2005).

La sabiduría de las multitudes se constituye de información suministrada en considerables cantidades y direcciones, cargadas mayormente de la opinión colectiva. Esta información conlleva a la generación de respuestas frente a situaciones particulares, sin embargo,

[...] la noción de la sabiduría de las multitudes no consiste sólo en que el grupo vaya a proporcionarnos siempre la respuesta exacta, sino que en promedio y a lo largo del tiempo aportará una respuesta mejor que la que cualquier individuo sería capaz de darnos (Surowiecki, 2005, p.203).

\section{Arquitectura de la participación}

La conceptualización acerca del término Web 2.0, por O'Reilly en 2004, introduce otros términos fundamentales ajustados a las cualidades de esta plataforma caracterizada por las interacciones sociales mediante múltiples instrumentos de cooperación, que asumen en el ciberespacio una estructura particular para la conformación de una red de colaboración entre individuos denominada, por el mismo autor, arquitectura de la participación.

Esta arquitectura de la participación emanada de la Web 2.0,

[...] brinda nuevas herramientas de empowerment [empoderamiento] $\mathrm{y}$, al mismo tiempo, de democratización en cuanto al intercambio del conocimiento. A fin de cuentas, todo este universo de desarrollos y avances 
tecnológicos tiene como pilar fundamental la valoración del usuario como pieza clave en el puzzle [rompecabezas] de la evolución tecnológica (Cobo, 2007a, p.49).

La arquitectura de la participación evidencia, más que un cambio tecnológico, un cambio social orientado a gestionar el conocimiento mediante una diversidad de herramientas que permiten tal acción.

\section{Servicios Web 2.0}

Los servicios web 2.0 son aquellos que permiten al usuario el acceso y la participación en los lugares del ciberespacio que cubran sus necesidades, estos pueden ser agrupados en cuatro ejes que contribuyen al desarrollo de la Internet:

a) Social Networking: describe todas aquellas herramientas diseñadas para la creación de espacios que promuevan o faciliten la conformación de comunidades e instancias de intercambio social. b) Contenidos: hace referencia a aquellas herramientas que favorecen la lectura y la escritura en línea, así como su distribución e intercambio. c) Organización Social e Inteligente de la Información: herramientas y recursos para etiquetar, sindicar e indexar, que facilitan el orden y almacenamiento de la información, así como de otros recursos disponibles en la Red. d) Aplicaciones y servicios (mashup): dentro de esta clasificación se incluye un sinnúmero de herramientas, softwares, plataformas en línea y un híbrido de recursos creados para ofrecer servicios de valor añadido al usuario final (Cobo, 2007b, p.63).

\section{Resultados}

En correspondencia con los objetivos del estudio, se aplicó la estadística descriptiva para desglosar datos importantes relacionados con los servicios web 2.0 en el portal de la UNICA orientados principalmente en plataformas digitales para redes sociales, esto debido a que el portal no presenta otro tipo de servicios como los de contenido, los de organización inteligente de la información o los de aplicaciones mashup. Los datos presentados en tablas surgen en función de la observación a las publicaciones realizadas desde las cuentas de usuario de la UNICA, durante 6 meses, en las plataformas YouTube, Instagram y SoundCloud (redes sociales verticales), así 
como en Facebook y Twitter (redes sociales horizontales). Las publicaciones denominadas historias en Instagram y Facebook se excluyen del estudio por lo efímero de su contenido (visible en la plataforma solamente por 24 horas).

En vista del comportamiento de cada plataforma (sus publicaciones, sus interacciones y otras particularidades), se consideró en este apartado las cuentas de usuario @ unicaeduve en Instagram y Twitter, plataformas que representan la red social vertical y la red social horizontal mayormente concurridas para establecer nexos con la comunidad universitaria.

Tabla Nº1. Número de publicaciones en la plataforma Instagram

\begin{tabular}{lcc}
\hline Publicaciones & f & \% \\
\hline enero & 36 & 28.1 \\
febrero & 19 & 14.8 \\
marzo & 20 & 15.6 \\
abril & 19 & 14.8 \\
mayo & 15 & 11.7 \\
junio & 19 & 14.8 \\
\hline Total & $\mathbf{1 2 8}$ & $\mathbf{1 0 0 \%}$ \\
\hline
\end{tabular}

Fuente: Elaboración propia

De acuerdo con los datos de la tabla $\mathrm{N}^{\circ} 1$, la cuenta de usuario @unicaeduve en la plataforma Instagram registró un total de 128 publicaciones durante el período de estudio. El mayor porcentaje de publicaciones se ubicó en el mes de enero, con 28.1\%, seguido de $15.6 \%$ en el mes de marzo. Las publicaciones del mes de mayo registraron el porcentaje más bajo con $11.7 \%$, mientras que un uniforme $14.8 \%$ se mantuvo en los meses restantes (febrero, abril y mayo). El mayor número de publicaciones coincide con un nuevo año académico para la comunidad universitaria, ciclo que inicia con los procesos de inscripción que comprenden los primeros lapsos académicos del año 2018 (LAC-2018 y LAR 2018-1), razón por la que los usuarios seguidores de la cuenta institucional requieren información precisa y detallada relacionada con diferentes procesos del calendario académico-administrativo de la UNICA. 
Tabla No2. Tipo de publicaciones en la plataforma Instagram

\begin{tabular}{lcc|lcc}
\hline Contenido & f & \% & Formato & f & \% \\
\hline Institucional & 85 & 66.4 & Imagen & 108 & 84.4 \\
Iglesia & 25 & 19.5 & Vídeo & 20 & 15.6 \\
Sociedad & 18 & 14.1 & - & & \\
\hline Total & $\mathbf{1 2 8}$ & $\mathbf{1 0 0 \%}$ & Total & $\mathbf{1 2 8}$ & $\mathbf{1 0 0 \%}$ \\
\hline
\end{tabular}

Fuente: Elaboración propia

En lo que respecta a la tabla $\mathrm{N}^{\circ} 2$, las publicaciones realizadas desde la plataforma Instagram fueron mayormente de tipo contenido institucional, con $66.4 \%$, las publicaciones contenido iglesia registraron un $19.5 \%$ y contenido sociedad un 14.1\%. Lo que revela que en la cuenta de usuario @ unicaeduve predomina información concerniente a la institución: fechas y procesos fijados en el calendario académicoadministrativo, autoridades universitarias, facultades y programas de estudio, entre otros de interés para la comunidad universitaria. El aspecto religioso católico también está presente en las publicaciones con importancia, no obstante, las publicaciones relacionadas con aspectos relevantes para la sociedad no reciben atención más allá de fechas obligatorias por algunos días festivos.

También, la tabla №2 discrimina las publicaciones de @unicaeduve, en Instagram, según el tipo de formato: el formato imagen obtuvo $84.4 \%$ y el formato vídeo el menor porcentaje con $15.6 \%$. La diferencia en puntos porcentuales entre ambos formatos es cónsona con el comportamiento que debe tener esta plataforma creada con el principal propósito de conformar redes sociales a partir de la difusión de publicaciones en formato imagen.

Tabla No3. Interacciones en la plataforma Instagram

\begin{tabular}{lcc}
\hline Interacción & f & \% \\
\hline Like & 13527 & 53.2 \\
Comentarios & 1521 & 6.0 \\
Reproducción de vídeos & 10400 & 40.9 \\
\hline Total & $\mathbf{2 5 4 4 8}$ & $\mathbf{1 0 0 \%}$ \\
\hline
\end{tabular}

Fuente: Elaboración propia

Las interacciones en la plataforma Instagram a través de la cuenta @ unicaeduve presentaron su mayor promedio, 53.2\%, en la opción like (me gusta), seguidamente la 
Rigo Grimaldos y Anny Paz

Telos Vol. 21, No. 3 (2019). 643-660

reproducción de vídeos publicados con 40.9\%, y apenas un 6\% registrado para los comentarios representó el menor promedio de interacción. Sin embargo, el total de interacciones generales no es significativo en proporción con el número de usuarios seguidores (alrededor de 12 mil), puesto que ese total engloba un período de seis meses y ligeramente duplica al número de seguidores. De realizar un análisis por cada unidad de publicación estas proporciones serían seriamente cuestionables.

Tabla N4. Número de publicaciones en la plataforma Twitter

\begin{tabular}{lcc}
\hline Publicaciones & f & \% \\
\hline enero & 219 & 21.0 \\
febrero & 177 & 17.0 \\
marzo & 195 & 18.7 \\
Abril & 159 & 15.2 \\
mayo & 118 & 11.3 \\
Junio & 175 & 16.8 \\
\hline Total & $\mathbf{1 0 4 3}$ & $\mathbf{1 0 0 \%}$ \\
\hline
\end{tabular}

Fuente: Elaboración propia

La tabla correspondiente al número de publicaciones (tweets) en la plataforma Twitter (tabla $\mathrm{N}^{\circ} 4$ ) esboza con detalle la distribución de 1043 publicaciones realizadas desde la cuenta de usuario @UNICAeduve. El mes de enero registró el mayor porcentaje de publicaciones con $21 \%$, seguido de un $18.7 \%$ del mes de marzo, mientras que el mes de mayo obtuvo $11.3 \%$ que lo ubicó como el mes con menos publicaciones. En un estadio intermedio febrero alcanza un $17 \%$, junio un $16.8 \%$ y abril un $15.2 \%$. Los resultados coinciden con el comportamiento de la institución frente a los procesos de inscripción que, durante el período de la investigación, involucra los lapsos académicos LAC-2018 (enero-marzo) y LAR 2018-1 (abril-julio). Durante el mes de junio inicia un nuevo ciclo de llamados para diferentes procesos, principalmente los de inscripción, correspondientes al segundo lapso académico regular del año (LAR 2018-2). 
Tabla $\mathbf{N}^{\circ} 5$. Tipos de publicaciones en la plataforma Twitter

\begin{tabular}{lcc|lcc}
\hline Contenido & $\mathbf{F}$ & $\mathbf{\%}$ & Formato & $\mathbf{f}$ & \% \\
\hline Institucional & 564 & 54.1 & Texto-Imagen & 820 & 78.6 \\
Iglesia & 257 & 24.6 & Texto-Vídeo & 27 & 2.6 \\
Sociedad & 65 & 6.2 & Texto & 196 & 18.8 \\
Respuesta & 157 & 15.1 & - & & \\
\hline Total & $\mathbf{1 0 4 3}$ & $\mathbf{1 0 0 \%}$ & Total & $\mathbf{1 0 4 3}$ & $\mathbf{1 0 0 \%}$ \\
\hline
\end{tabular}

Fuente: Elaboración propia

El tipo de publicaciones realizadas desde la plataforma Twitter, registradas en la tabla $\mathrm{N}^{\circ} 5$, concentra la mayoría de publicaciones en contenido institucional con $54.1 \%$, un $24.6 \%$ alcanza el contenido iglesia seguido del contenido respuesta con $15.1 \%$, finalmente el contenido sociedad se posiciona en el último lugar con el menor número de publicaciones, 6.2\%. Evidentemente, los contenidos con temática sociedad no representan una prioridad para la cuenta de usuario @UNICAeduve que centra su foco en informar sobre el acontecer intrauniversidad, las publicaciones contenido respuesta hacen presencia como publicaciones de acuerdo con las características de la plataforma Twitter.

Por otro lado, la tabla N5 también registra las publicaciones según el tipo de formato, las publicaciones de formato texto-imagen alcanzan un $78.6 \%$, el formato texto $18.8 \%$ y el formato texto-vídeo $2.6 \%$ en última posición. De acuerdo con las particularidades de la plataforma Twitter, las publicaciones con mayor interacción o efectividad acostumbran estar acompañadas en combinación texto-imagen o textovídeo, lo que indica que en @UNICAeduve existe un uso adecuado de los formatos al momento de realizar un tweet. La cantidad de tweets y la frecuencia de cada publicación dejan en evidencia el uso de aplicaciones para gestionar este tipo de plataformas.

\begin{tabular}{|c|c|c|}
\hline Interacción & f & $\%$ \\
\hline Reproducción de vídeos & 2328 & 33.5 \\
\hline Retweet & 2516 & 36.2 \\
\hline Like & 2109 & 30.3 \\
\hline Total & 6953 & $100 \%$ \\
\hline
\end{tabular}

Fuente: Elaboración propia 
La tabla $\mathrm{N}^{\circ} 6$ presenta las interacciones en la plataforma Twitter desde la cuenta de usuario de la UNICA. El retweet alcanzó el primer lugar con 36.2\%, la reproducción de vídeos el segundo con $33.5 \%$ y el like el último lugar con 30.3\%. La diferencia en puntos porcentuales entre cada tipo de interacción no es realmente distintiva, esto probablemente se debe a que la estructura y funcionamiento de la plataforma permite a los usuarios realizar las tres interacciones casi de forma simultánea. No obstante, es importante destacar que el número total de interacciones no es cónsono en relación con el número de usuarios seguidores que está ubicado alrededor de 18 mil, la cantidad de interacciones es realmente baja.

\section{Conclusiones}

El portal de la Universidad Católica Cecilio Acosta (www.unica.edu.ve) presenta en su cabecera cinco (5) botones-íconos que direccionan a los visitantes hacia algunas plataformas digitales para redes sociales: Facebook, Twitter, Instagram, SoundCloud y YouTube; plataformas en las que la UNICA posee cuentas de usuario e interactúa con su comunidad universitaria. Por otro lado, el portal no comparte servicios como plataformas de contenido, organización inteligente de información y aplicaciones mashup; quizás el botón que vincula a la página con la plataforma educativa Moodle (UNICAdis Virtual) podría ser una aproximación a plataformas de contenido, pero su acceso es limitado (estudiantes y docentes de la modalidad virtual). Los servicios web 2.0 en el portal de la UNICA se concentran en el uso específico de aplicaciones para redes sociales (social networking).

Las plataformas digitales para redes sociales Instagram y Twitter son las que la UNICA utiliza con mejor eficacia para entrelazar redes sociales y difundir contenido mayormente informativo referido a la universidad y otros temas relacionados con la iglesia católica, lo poco publicado inherente a temas de la sociedad se limita a fechas forzosas en la cultura venezolana. La cantidad de interacciones con sus usuarios no es representativa en relación con la cantidad de seguidores en ambas plataformas. La 
plataforma Facebook, aunque es usada por la UNICA y posee un considerable número de publicaciones, genera una interacción casi imperceptible. Los canales en las plataformas SoundCloud y YouTube no reciben el uso pertinente según las características de los formatos de contenido que permite cada una. Las respuestas emitidas por el Community Manager de la UNICA a los comentarios en cualquiera de las redes conformadas son oportunas y enmarcadas en la institucionalidad. El uso de los servicios web 2.0 en el portal de la UNICA por parte de la institución es limitado y poco estratégico.

La Web 2.0 como evolución de la denominada World Wide Web pretende consolidar la visión de su creador, Berners-Lee, al generar servicios que suman a la interactividad un componente social que conlleva a la materialización de redes de gestión del conocimiento, en las cuales la transferencia de datos implica procesos sociales de intercambio y de construcción colectiva. En este sentido, la UNICA al presentar el uso de servicios web 2.0 antes señalado limita los procesos de gestión del conocimiento que deben emerger en las organizaciones universitarias del siglo XXI.

Se recomienda a la UNICA dirigir el contenido de sus cuentas en plataformas para redes sociales a un público más amplio, ir más allá de la comunidad estudiantil y acercarse a la comunidad universitaria en general, a las comunidades aledañas, a los ciudadanos en la ciudad del Maracaibo y sus fronteras, con el propósito de fortalecer el vínculo esencial de la universidad con la sociedad: la construcción plural de saberes. Además, se sugiere actualizar el portal web de la institución y agregar servicios de contenido, de organización inteligente de información y de aplicaciones mashup, los mismos contemplados en la educación del nuevo siglo para la construcción del conocimiento.

\section{Referencias Bibliográficas}

Berners-Lee, Tim. (2000). Tejiendo la red. El inventor del World Wide Web nos descubre su origen. Siglo XXI Editores. España.

Cabero, Julio. (2009). Educación 2.0. ¿Marca, moda o nueva visión de la educación? En Castaño Carlos (Coord.), Web 2.0. El uso de la web en la sociedad del 
conocimiento (pp. 13-34) (Ebook). Universidad Metropolitana. Venezuela. Extraído de:

https://www.researchgate.net/profile/Julio_Almenara/publication/236856422_Web _20_El_uso_de_la_web_en_la_sociedad_del_conocimiento/links/02e7e519891bde 0b2f000000/Web-20-El-uso-de-la-web-en-la-sociedad-del-conocimiento.pdf consulta: 25/3/2016.

Castaño, Carlos; Maíz, Inmaculada; Palacio, Gorka y Domingo, José. (2008). Prácticas educativas en entornos 2.0 (Ebook). Editorial Síntesis SA. España. Extraído de: https://www.sintesis.com/tecnologia-educativa-73/practicas-educativas-enentornos-web-2-0-ebook-1224.html consulta: 4/3/2016.

Cobo, Cristóbal. (2007a). Intercreatividad y Web 2.0. La construcción de un cerebro digital planetario. En Cobo Cristóbal y Pardo Hugo, Planeta Web 2.0. Inteligencia colectiva o medios fast food (pp. 43-60) (Ebook). Grup de Recerca d'Interaccions Digitals (Universitat de Vic) y Flacso México. España / México. Extraído de: https://books.google.com.uy/books?id=ptMCLfJTSxEC\&printsec=frontcover\&hl= es consulta: $20 / 8 / 2017$.

Cobo, Cristóbal. (2007b). Mapa de aplicaciones. Una taxonomía comentada. En Cobo Cristóbal y Pardo Hugo, Planeta Web 2.0. Inteligencia colectiva o medios fast food (pp. 61-88) (Ebook). Grup de Recerca d'Interaccions Digitals (Universitat de Vic) y Flacso México. España / México. Extraído de: https://books.google.com.uy/books?id=ptMCLfJTSxEC\&printsec=frontcover\&hl= es consulta: $20 / 8 / 2017$.

Drucker, Peter. (2004). La sociedad postcapitalista. Grupo Editorial Norma. Colombia.

García, Fernando. (2010). Internet en la vida de nuestros hijos: ¿Cómo transformar los riesgos en oportunidades? (Ebook). Foro Generaciones Interactivas. España. Extraído de: https://issuu.com/generacionesinteractivas/docs/internet_en_la_vida_de_nuestros hijos consulta: 15/5/2016.

Ibeas, Ángel; Díaz, José y De La Hoz, Daniel. (2000). E- logistics (I): Nuevas tecnologías de la información (I-net). Centro Intermodal de Logística SA (CILSA), Instituto de Logística Interamericano (ILI) y Marge Design Editors SL. España.

Johnson, Steve. (2001). Emergence. The connected lives of ants, brains, cities and software. Penguin books. England.

Lévy, Pierre. (2004). Inteligencia colectiva: por una antropología del ciberespacio (Ebook). Organización Panamericana de la Salud (OPS). Estados Unidos. Extraído de:

http://inteligenciacolectiva.bvsalud.org/public/documents/pdf/es/inteligenciaColect iva.pdf consulta: 10/6/2017.

Nafría, Ismael. (2008). La web 2.0: El usuario el nuevo rey de Internet (cuarta edición). Ediciones Gestión 2000. España. 
O' Reilly, Tim. (2007). What is Web 2.0? (Entrevista Kamla Bhatt) [Grabación canal YouTube]. Extraído de: https://www.youtube.com/watch?v=CQibri7gpLM consulta: 5/2/2016.

Rheingold, Howard. (2004). Multitudes inteligentes: La próxima revolución. Editorial Gedisa. España.

Rojo, Guillermo y Sánchez, Mercedes. (2009). El español en la red (Ebook). Editorial Ariel, SA y Fundación Telefónica. España. Extraído de: https://www.fundaciontelefonica.com/arte_cultura/publicaciones-listado/paginaitem-publicaciones/itempubli/5/ consulta: 8/5/2016.

Surowiecki, James. (2005). Cien mejor que uno. La sabiduría de la multitud o por qué la mayoría siempre es más inteligente que la minoría. Ediciones Urano. España. 